\title{
Eosinophils are more relevant than basophils to allergic sensitization in pediatric adenotonsillar hypertrophy
}

\author{
Juanjuan Zou ${ }^{1}$, Yan Yang ${ }^{1}$, Qiang $\mathrm{Fu}^{1}$, Huayang $\mathrm{Liu}^{1}$, Yan Wang ${ }^{1}$, and Yanzhong $\mathrm{Li}^{1}$ \\ ${ }^{1}$ Shandong University Qilu Hospital
}

June 24, 2020

\begin{abstract}
Objective: Eosinophils and basophils both play important roles in the immune response, but the relationships between them and allergy in adenotonsillar hypertrophy (ATH) children is unclear. This study aimed to investigate the relationship between eosinophil/basophil counts and peripheral specific IgE levels, and identify the common allergens in children with ATH. Methods: We initially screened 931 consecutive children who underwent adenotonsillectomy in our department from 2018 to 2019 , and finally included 676 children. The eosinophil count, basophil count, and specific IgE levels were evaluated. The eosinophil count, basophil count and levels of specific IgE were collected. Logistic regression analyses were performed to evaluate the odds ratios (ORs) and $95 \%$ confidence intervals (95\% CIs) for atopy. Results: The eosinophil and basophil counts in atopic participants were significantly higher compared to non-atopic participants. The eosinophil count correlated with the levels of IgE specific to all allergens, and eosinophilia was independently associated with all tested atopy allergens other than atopy to dander after multivariate adjustment for age, sex, vitamin D, BMI, and visiting season. Additionally, the basophil count correlated with the IgE levels specific to A. alternate and food mix, and basophilia was significantly associated with atopy to food mix after multivariable adjustment. Furthermore, among allergic participants, D. farinae was the most prevalent allergen, followed by food mix, D. pteronyssinus and A. alternata. Conclusion: Eosinophils were more relevant to allergic sensitization than basophils, with eosinophils being significantly associated with all tested atopy allergens apart from dander, and basophils being associated with atopy to food mix. Furthermore, D. farinae was the most prevalent allergen and may be indicative of desensitization therapy.
\end{abstract}

\section{Hosted file}

Main_manuscript.docx available at https://authorea.com/users/336520/articles/462237eosinophils-are-more-relevant-than-basophils-to-allergic-sensitization-in-pediatricadenotonsillar-hypertrophy

\section{Hosted file}

Figures.docx available at https://authorea.com/users/336520/articles/462237-eosinophilsare-more-relevant-than-basophils-to-allergic-sensitization-in-pediatric-adenotonsillarhypertrophy 